TRANSACTIONS OF THE

AMERICAN MATHEMATICAL SOCIETY

Volume 286, Number 2, December 1984

\title{
THE GODBILLON-VEY INVARIANT OF A TRANSVERSELY HOMOGENEOUS FOLIATION
}

BY

\author{
ROBERT BROOKS AND WILLIAM GOLDMAN ${ }^{1}$
}

\begin{abstract}
A real projective foliation is a foliation $\mathfrak{F}$ with a system of local coordinates transverse to $\mathfrak{F}$ modelled on $\mathbf{R} P^{1}$ (so that the coordinate changes are real linear fractional transformations). Given a closed manifold $M$, there is but a finite set of values in $H^{3}(M ; \mathbf{R})$ which the Godbillon-Vey invariant of such foliations may assume. A bound on the possible values, in terms of the fundamental group, is computed. For $M$ an oriented circle bundle over a surface, this finite set is explicitly computed.
\end{abstract}

In [5], C. Godbillon and J. Vey introduced a characteristic class associated to a codimension-one foliation which lies in the third real cohomology group of the ambient manifold. The question was then raised whether one could assign some geometric significance to this invariant. The problem of understanding the GodbillonVey invariant in such a manner was underscored by a result of Thurston [12], who showed that for any closed 3-manifold there are families of codimension-one foliations whose Godbillon-Vey invariant varies continuously in a parameter, and which take on arbitrarily large values.

It is the main goal of this paper to show that this situation changes when one restricts to foliations which are transversely homogeneous (see §1). To explain the geometric picture which emerges, it is worthwhile reviewing briefly Gromov's notion of simplicial volume [7].

Given a compact manifold $M$, Gromov assigns to $M$ a volume $\|M\|$, defined topologically, which measures the complexity of representing the fundamental class of $M$ by singular simplices. For many manifolds, for instance spheres, it is easily seen that $\|M\|=0$. However, inspired by the theorem of Milnor [9] and Wood [15], Gromov and Thurston (see [7]) were able to show that when $M$ is a hyperbolic manifold, $\|M\|$ is equal to the volume of $M$ in its hyperbolic metric, up to a constant depending only on $\operatorname{dim}(M)$. This is done in part by comparing $\|M\|$ to a certain volume invariant associated to representations from $\pi_{1}(M)$ to the isometry group of hyperbolic space. At the heart of the argument is the elementary fact that a geodesic simplex in hyperbolic space has bounded volume.

In the present case, we consider foliations of a manifold $M$ which are transversely projective, that is, which are locally modelled on the action of $\operatorname{PSL}(2, \mathbf{R})$ on the circle $S^{\mathbf{1}}$. The holonomy of such a foliation is described by a homomorphism $\pi_{1}(M) \rightarrow(\operatorname{SL}(2, \mathbf{R}))^{\sim}$, the universal covering of $\operatorname{PSL}(2, \mathbf{R})$, as is discussed in $\S \S 1$ and 2 .

Received by the editors September 13, 1983 and, in revised form, December 8, 1983.

1980 Mathematics Subject Classification. Primary 53C10, 53C12, 57R32, 55R40.

${ }^{1}$ The authors research was partially supported by NSF grants. $0002-9947 / 84 \$ 1.00+\$ .25$ per page 
We then show in $\S \S 2$ and 3 how the Godbillon-Vey invariant is naturally a volume invariant of this homomorphism, and so is expressible, via the machinery of continuous cohomology, in terms of volumes of "geodesic simplices" in $(\operatorname{SL}(2, \mathbf{R}))^{\sim}$. We also show that this invariant is rigid under deformations, in contrast to Thurston's examples [12].

In $\S \S 4-6$, we use these results to show that for a fixed manifold $M$ the GodbillonVey invariant takes on only finitely many values as the foliation ranges over all transversely projective foliations of $M$, and we produce an explicit topological bound for the largest value. To explain this topological bound, we first observe that a geodesic simplex in $(\operatorname{SL}(2, \mathbf{R}))^{\sim}$ need not have bounded volume. However, we show in $\S 4$ that the volume of such a geodesic simplex is bounded in terms of a certain "winding invariant", which measures how much an element of $(\operatorname{SL}(2, \mathbf{R}))^{\sim}$ rotates the circle $S^{1}$. The topological bound is then analogous to Gromov's construction, except that each simplex is weighted according to this winding invariant. We then show in $\S \S 5$ and 6 how to modify a representation. $\pi_{1}(M) \rightarrow(\operatorname{SL}(2, \mathbf{R}))^{\sim}$ to keep the winding numbers small. This is, in effect, a variation of the argument of Wood [15].

It is this weighting by the winding number which gives this topological bound new and interesting features.

In $\S \S 6$ and 7 , we then work out these bounds explicitly for circle bundles with Euler class $e \neq 0$ over surfaces, with Euler characteristic $\chi(S)<0$. We find that for $\mathfrak{F}$ a real projective foliation,

$$
|G-V(\mathfrak{F})| \leq\left(4 \pi^{2}\right)\left|\chi^{2}(S) / e\right|
$$

The right-hand side has an obvious interpretation in the setting of Thurston's eight geometries (see [21])-namely, it is the volume of such spaces in the geometric structure applicable to nontrivial circle bundles over surfaces, which is Geometry \#6 on Thurston's list [21].

This is the geometric interpretation of the Godbillon-Vey invariant we seek. In particular, the Godbillon-Vey invariant allows us to talk about the volume of a 3-manifold in this geometry when the manifold does not have this geometry-for instance, if the manifold is hyperbolic.

In a subsequent paper [20], we will elaborate more fully on these results in the context of 3-manifolds and of Thurston's eight geometries. It will be shown there that the Godbillon-Vey invariant, suitably interpreted, becomes an interesting invariant in the study of hyperbolic 3-manifolds.

In [8], Heitsch has recently extended our rigidity and finiteness results to some transversely homogeneous foliations of higher codimension.

We would like to thank Walter Neumann for carefully reading this manuscript and suggesting several improvements.

1. Transverse structures on foliations. Let $M$ be a smooth manifold. A smooth codimension- $k$ foliation $\left\{U_{\alpha}\right\}$ of $M$ is defined by a coordinate chart on $M$, together with submersions $\varphi_{\alpha}: \mathcal{U}_{\alpha} \rightarrow \mathbf{R}^{k}$, such that for each overlap $\mathcal{U}_{\alpha} \cap \mathcal{U}_{\beta}$, there is a local diffeomorphism $g_{\alpha \beta}$ of $\mathbf{R}^{k}$ such that

$$
\varphi_{\alpha}=g_{\alpha \beta} \circ \varphi_{\beta}
$$

An infinitesimal characterization of a foliation is given by the theorem of Frobenius - a codimension- $k$ foliation is described by a codimension- $k$ subbundle of the 
tangent bundle of $M$ which is integrable, i.e., such that its sections are closed under the Lie bracket. In the language of differential forms, this integrability condition is easily expressed: a codimension- $k$ foliation is locally given by $k$ everywhere linearly independent 1 -forms $\varphi_{1}, \ldots, \varphi_{k}$, such that

$$
d \varphi_{i}=\sum \omega_{i j} \wedge \varphi_{j}
$$

for some 1-forms $\omega_{i j}$. The tangent space to the foliation is then the simultaneous kernel of the $\varphi_{i}$ 's-we say that the $\varphi_{i}$ 's define $\mathfrak{F}$ by this recipe. (For more general background on foliations, see [16].)

We may obtain finer structures on foliations by imposing special conditions on the transition functions $g_{\alpha \beta}$. If $X$ is a $k$-dimensional manifold, and $G$ a group of diffeomorphisms of $X$, we say that $\mathfrak{F}$ has a transverse $(G, X)$ structure if the $\varphi$ 's can be taken to be submersions onto $X$, and if the $g_{\alpha \beta}$ 's can be taken to lie in $G$.

An important special case is when $G$ is a Lie group and $X$ a homogeneous space of $G$. In this case, we call $\mathfrak{F}$ a transversely homogeneous space. For instance when $k=1$, we have the following types of transverse structures:

(a) $G=$ the group of Euclidean translations of $\mathbf{R}$. $\mathfrak{F}$ is then called a transversely Euclidean foliation.

(b) $G=$ the group of affine translations of $\mathbf{R}$. $\mathfrak{F}$ is then a transversely affine foliation.

(c) $G=$ the group $\operatorname{PSL}(2, \mathbf{R})$ of projective transformations of $\mathbf{R} P^{1}$. $\mathfrak{F}$ is a transversely projective or $\mathbf{R} P^{1}$-foliation.

The case (c) will be the primary focus of this paper. In [1], Blumenthal has given a differential form characterization of transversely homogeneous structures. We give it first for cases (a)-(c) above, and then refer to [1] for a general formulation.

Proposition 1. (a) A foliation is transversely Euclidean if and only if it is defined by a closed 1-form.

(b) A foliation is transversely affine if and only if there are global 1-forms $\omega, \theta$ such that:

(i) $\omega$ defines $\mathfrak{F}$;

(ii) $d \omega=\theta \wedge \omega, d \theta=0$.

(c) $A$ foliation has an $\mathbf{R} P^{1}$-structure if and only if there are 1 -forms $\omega, \theta, \eta$ such that:

(i) $\omega$ defines $\mathfrak{F}$;

(ii) $d \omega=\theta \wedge \omega, d \theta=\omega \wedge \eta$ and $d \eta=\eta \wedge \theta$.

We recognize in (a)-(c) the Maurer-Cartan equations of the corresponding Lie groups. A general characterization of transversely homogeneous foliations in terms of differential forms is carried out in [1], to which the reader is referred for details.

We close this section with some basic examples. Suppose $M$ is a manifold and $h: \pi_{1}(M) \rightarrow G$ a homomorphism. If $X$ is a homogeneous space for $G$ (or more generally any space on which $G$ acts), we may form the flat bundle $(X) M$ with fiber $X$ determined by $h$. The flat structure on $(X) M$ is equivalent to a foliation on $(X) M$ transverse to the fibers, and it is easily seen that this is a transverse $(G, X)$-foliation.

Furthermore, any flat $G$-bundle over $M$ is uniquely determined by such a homomorphism $h: \pi_{1}(M) \rightarrow G$, called the holonomy homomorphism. (See [18] for generalities about flat bundles.) 
If $\mathfrak{F}$ is a transversely homogeneous foliation of a manifold $N$, and $\mathfrak{f}: M \rightarrow N$ is a map transverse to the foliation, then $\mathfrak{f}^{*}(\mathfrak{F})$ defines a foliation on $M$ which is again transversely homogeneous.

In general, this gives rise to new transverse homogeneous foliations which are not flat $G$-bundles. For instance, let $E$ be a circle bundle over a Riemann surface $S$ of genus $g$, with Euler class $e(E)$. Then, by a theorem of Milnor [9] and Wood [15], $E$ carries a flat $\operatorname{PSL}(2, \mathbf{R})$-structure if and only if $|e(E)| \leq|\chi(S)|$.

However, taking an $|e(E)|$-fold covering of the fiber gives an $|e(E)|$-fold covering of $E$, which is now a circle bundle over $S$ with Euler class 1 . It is not hard to see that this new foliation is no longer given by a flat $\operatorname{PSL}(2, \mathbf{R})$-bundle over $S$, although it remains transversely $\operatorname{PSL}(2, \mathbf{R})$-homogeneous.

On the other hand, the following construction shows that all tranversely homogeneous foliations arise from flat $G$-bundles in a natural way: Suppose that $\mathfrak{F}$ is a transverse $(G, X)$-foliation of $M$. We will construct a flat $G$-bundle $(X) M$ over $M$, with fiber $X$, in the following way: over each neighborhood $\mathcal{U}_{\alpha} \subset M$, we form the product $U_{\alpha} \times X$, which is given the product foliation defined by projection onto the second factor.

The overlap transformation

$$
\left(\mathcal{U}_{\alpha} \cap \mathcal{U}_{\beta}\right) \times X \subset \mathcal{U}_{\alpha} \times X \rightarrow \mathcal{U}_{\beta} \times X
$$

given by $(u, x) \rightarrow\left(u, g_{\alpha \beta}(x)\right)$, allows us to glue these neighborhoods together to obtain a bundle $(X) M$. Since the overlap transformations evidently preserve the product foliation, this foliation defines the flat structure on $(X) M$. Let $\mathfrak{F}^{\prime}$ denote this foliation.

The holonomy homomorphism $h: \pi_{1}(M) \rightarrow G$ which determines this flat bundle is called the holonomy homomorphism of $\mathfrak{F}$.

The following lemma will be extremely useful in what follows.

LEMMA 1 . There is a canonically defined section $\delta: M \rightarrow(X) M$, called the developing section, such that $\delta$ is transverse to $\mathfrak{F}^{\prime}$ and $\delta^{*}\left(\mathfrak{F}^{\prime}\right)=\mathfrak{F}$.

In particular, this shows that all transversely homogeneous foliations are "deduced" from flat bundles.

Proof. We define $\delta$ on each neighborhood $U_{\alpha} \times X$ to be the graph of the submersion $\Phi_{\alpha}: \mathcal{U}_{\alpha} \rightarrow X$. The equation $\varphi_{\alpha}=g_{\alpha \beta} \circ \varphi_{\beta}$ shows that $\delta$ glues together to give a global section of the bundle $(X) M$. The fact that $\delta$ is transverse to $\mathfrak{F}$ is then equivalent to the fact that the $\varphi_{\alpha}$ 's are submersions.

Note that when we pull back the flat bundle $(X) M$ to the universal cover $\tilde{M}$ of $M$, the induced bundle $(X) M$ is canonically trivial. The developing section $\tilde{\delta}$ then lifts to a submersion $\tilde{\delta}: \tilde{M} \rightarrow X$. This is the analogue of the developing map for geometric structures (see, e.g., $[6]$ ).

2. The Godbillon-Vey invariant. Let $\mathfrak{F}$ be a transversely oriented codimension-one foliation. The Frobenius integrability condition then reduces to the assertion that, for $\varphi$ a defining 1-form for $\mathfrak{F}$, there is a 1 -form $\theta$ such that $d \varphi=\theta \wedge \varphi$.

It was observed by Godbillon and Vey [5] that the form $\theta \wedge d \theta$ is closed, and that its cohomology class depends only on $\mathfrak{F}$. This cohomology class is called the Godbillon-Vey invariant of $\mathfrak{F}$. 
Note that if $f: M \rightarrow N$ is transverse to a foliation $\mathfrak{F}$ on $N$, then $G-V\left(f^{*}(\mathfrak{F})\right)=$ $f^{*}(G-V(\mathfrak{F}))$.

It is easily seen from Proposition 1 that the Godbillon-Vey class of an affine foliation is $\mathbf{0}$. However, for $\mathbf{R} P^{\mathbf{1}}$-foliations the situation is different. The following proposition can be found in [17]-it also follows readily from results in $\S 3$.

Proposition 2. Suppose that

$$
\begin{gathered}
E \\
\downarrow \\
M
\end{gathered}
$$

is a flat PSL(2, R)-bundle over $M$ with fiber $S^{1}$. Then integrating $G-V(\mathfrak{F})$ over the fiber $S^{1}$ gives

$$
\int_{S^{1}} G-V(\mathfrak{F})=\left(4 \pi^{2}\right) e(E)
$$

where $e(E) \in H^{2}(M ; \mathbf{R})$ is the Euler class of this bundle $E$. (Here, $\mathfrak{F}$ is the foliation transverse to the fibers given by the flat structure of $E$.)

In particular, $G-V$ may be nonzero.

In [12], Thurston gave examples of foliations varying smoothly in a parameter, such that the Godbillon-Vey invariant varies smoothly over $\mathbf{R}$. (See $[3]$ for a more complete discussion.) Our first result shows that this cannot happen for $\mathbf{R} P^{1}$. foliations:

THEOREM 1 (RIGIDITY THEOREM). Let $\mathfrak{F}_{t}$ be a family of $\mathbf{R} P^{1}$-foliations of a manifold $M$ varying smoothly in $t$. Then $G-V\left(\mathfrak{F}_{t}\right)$ is constant for all $t$.

ProOF. By Proposition 1(c), we may assume that there are 1-forms $\omega_{t}, \theta_{t}$ and $\eta_{t}$ varying smoothly in $t$, satisfying conditions (i) and (ii).

For convenience, we label these conditions
(A) $d \omega_{t}=\theta_{t} \wedge \omega_{t}$
(B) $d \theta_{t}=\omega_{t} \wedge \eta_{t}$
(C) $d \eta_{t}=\eta_{t} \wedge \theta_{t}$.

From (A) and (B), it follows that

$$
G-V\left(\mathfrak{F}_{t}\right)=\omega_{t} \wedge \eta_{t} \wedge \theta_{t}
$$

Denoting by $\cdot$ differentiation with respect to $t$, it suffices to show that $(G \dot{-} V)$ is exact. But

$$
(G \dot{-V})=\dot{\omega} \wedge \eta \wedge \theta+\omega \wedge \dot{\eta} \wedge \theta+\omega \wedge \eta \wedge \dot{\theta}
$$

and differentiating $(\mathrm{A})-(\mathrm{C})$ gives

$\left(\mathrm{A}^{\prime}\right) d \dot{\omega}=\dot{\theta} \wedge \omega+\theta \wedge \dot{\omega}$,

(B') $d \dot{\theta}=\dot{\omega} \wedge \eta+\omega \wedge \dot{\eta}$

$\left(\mathrm{C}^{\prime}\right) d \dot{\eta}=\dot{\eta} \wedge \theta+\eta \wedge \dot{\theta}$.

Applying $(\mathrm{A})-(\mathrm{C})$ to the undifferentiated terms of $(*)$ gives

$$
(G \dot{-} V)=\dot{\omega} \wedge d \eta+\dot{\eta} \wedge d \omega+\dot{\theta} \wedge d \theta
$$

while applying $\left(\mathrm{A}^{\prime}\right)-\left(\mathrm{C}^{\prime}\right)$ gives

$$
2(G \dot{-} V)=d \dot{\theta} \wedge \theta+d \dot{\eta} \wedge \omega+d \dot{\omega} \wedge \eta
$$


and adding $(* *)$ and $(* * *)$ gives

$$
3(G-V)=d(\dot{\omega} \wedge \eta+\dot{\eta} \wedge \omega+\dot{\theta} \wedge \theta)
$$

which is the desired result.

Theorem 1 is a special case of a more general rigidity theorem for semisimple Lie groups, which we now state. We are grateful to David Vogan for insight into its proof. To state it, let $\mathfrak{g}$ be a semisimple Lie algebra, $\mathfrak{g}^{*}$ its dual Lie algebra, and $A^{*}(\mathfrak{g})$ the exterior algebra on $\mathfrak{g}^{*}$. When $\mathfrak{g}$ is the Lie algebra of $G$, then $A^{*}(\mathfrak{g})$ is the complex of left-invariant forms on $G$.

A $\mathfrak{g}^{*}$-structure on $M$ is a map $A^{*}(\mathfrak{g}) \rightarrow A^{*}(M)$ which commutes with $d$ and with wedge product.

THEOREM 2. Let $F_{t}: A^{*}(\mathfrak{g}) \rightarrow A^{*}(M)$ be a family of $\mathfrak{g}^{*}$-structure on $M$. If $\mathfrak{g}$ is semisimple, the induced map on cohomology is independent of $t$.

Proof. Denote by $V$ the coadjoint $\mathfrak{g}$-module, i.e. $V=\mathfrak{g}^{*}$ with the module structure contragradient to the adjoint representation. Let $\left\{\tilde{\omega}_{1}, \ldots, \tilde{\omega}_{m}\right\}$ be a basis of $\mathfrak{g}^{*}$, and $\left\{\omega_{1}, \ldots, \omega_{m}\right\}$ the corresponding basis of $V$. As above, we must show that $d F / d t$ is zero in cohomology. But $d F / d t$ factors into

$$
d F / d t: A^{*}(\mathfrak{g}) \stackrel{J}{\rightarrow} A^{*-1}(\mathfrak{g}, V) \stackrel{\dot{F}}{\rightarrow} A^{*}(M),
$$

where $J$ is given by

$$
J\left(\tilde{\omega}_{i_{1}} \wedge \cdots \wedge \tilde{\omega}_{i_{k}}\right)=\sum_{j=1}^{k}(-1)^{j} \tilde{\omega}_{i_{1}} \wedge \cdots \wedge \tilde{\omega}_{i_{j}} \wedge \cdots \wedge \tilde{\omega}_{i_{k}} \otimes \omega_{i_{j}}
$$

and $\dot{F}$ is given by

$$
\dot{F}(\xi \otimes \omega)=F(\xi) \wedge(d \omega / d t)
$$

On the other hand, the semisimplicity of $\mathfrak{g}$ implies already that $H^{*}(\mathfrak{g} ; V)=0$ (see $[\mathbf{1 9}]$ ) so that $d F / d t$ must be zero in cohomology.

The rigidity theorem for $\operatorname{PSL}(2, \mathbf{R})$ yields readily a finiteness theorem, Theorem 3 below. The technique is a variant of one given by Sullivan [10].

THEOREM 3 (FINITENESS). Let $M$ be a manifold with finitely generated fundamental group. Then the set of Godbillon-Vey invariants of $\mathbf{R} P^{1}$-foliations of $M$ is finite.

PROOF. Suppose that $\mathfrak{F}$ is an $\mathbf{R} P^{1}$-foliation, and let $h: \pi_{1}(M) \rightarrow \operatorname{PSL}(2, \mathbf{R})$ be its holonomy representation.

LEMMA 2. $G-V(\mathfrak{F})$ depends only on the holonomy representation $h$.

PROOF. The foliation $\mathfrak{F}$ is determined by two pieces of data: the flat circle bundle $\left(S^{1}\right) M$ constructed from $h$, and the developing section $\delta$, as in Lemma 1 . Let $\tilde{\delta}$ be any trivialization of $\left(S^{1}\right) M$. By the Kunneth theorem, we may use $\tilde{\delta}$ to identify $H^{*}\left(\left(S^{1}\right) M\right)$ with $H^{*}(M) \otimes H^{*}\left(S^{1}\right)$. In general, we would expect $G-V$ to have two components - a $(3,0)$-component and a $(2,1)$-component.

However, the $(2,1)$-component, integrated over the fiber, must be proportional to the Euler class, by Proposition 2, and so is zero, since $\left(S^{1}\right) M$ is topologically 
trivial. The identification of the $(3,0)$-component with a class in $H^{3}(M ; \mathbf{R})$ is given by the projection map, and so does not depend on the choice of section $\varepsilon$.

Now the set of homomorphisms $\pi_{1}(M) \rightarrow \operatorname{PSL}(2, \mathbf{R})$ forms a real algebraic variety, and so has only finitely many components, by a theorem of Whitney. On each component, the Godbillon-Vey invariant on $\left(S^{1}\right) M$ is constant by the rigidity theorem. If we now restrict our attention to those components for which $\left(S^{1}\right) M$ has a section, then Lemma 2 shows that the Godbillion-Vey invariant of $M$ must be constant on each of these components. It follow that there are only finitely many possible values for $G-V(\mathfrak{F})$, yielding Theorem 3 .

3. Continuous cohomology. Let $G$ be a Lie group. If we regard $G$ as a discrete group, then its group cohomology is computed by cohomology of the Eilenberg-Mac Lane complex, whose $k$-cochains are given by

$$
C^{k}(G)=\{f: G \underbrace{\times \cdots \times}_{k \text { times }} G \rightarrow \mathbf{R}\}
$$

and where $\delta: C^{k}(G) \rightarrow C^{k+1}(G)$ is given by

$$
\begin{aligned}
\delta f\left(g_{0}, \ldots, g_{k}\right)= & f\left(g_{1}, \ldots, g_{k}\right)+\sum_{i}(-1)^{i} f\left(g_{0}, \ldots, g_{i} g_{i-1}, \ldots, g_{k}\right) \\
& +(-1)^{k+1} f\left(g_{0}, \ldots, g_{k-1}\right) .
\end{aligned}
$$

Denote by $H_{E-M}^{*}(G ; \mathbf{R})$ the cohomology of this complex. $H_{E-M}^{*}(G ; \mathbf{R})$ represents the set of characteristic classes of flat $G$-bundles-namely, if there is a representation $\pi_{1}(M) \rightarrow G$, then there is a natural map $H_{E-M}^{*}(G ; \mathbf{R}) \rightarrow H^{*}(M ; \mathbf{R})$ "classifying" this representation.

For a general lie group, of course, $H_{E-M}^{*}(G ; \mathbf{R})$ is an enormously complicated space, and in any practical sense is completely unwieldy to work with. It is convenient to substitute for this complex the continuous subcomplex

$$
C_{\text {cont }}^{k}(G)=\{f: G \times \cdots \times G \rightarrow \mathbf{R} \text { such that } f \text { is continuous }\}
$$

which is clearly preserved by $\delta$. The resulting cohomology is the continuous cohomology of $G, H_{\text {cont }}^{*}(G ; \mathbf{R})$. Then $H_{\text {cont }}^{*}(G ; \mathbf{R})$ consists of characteristic classes of representations $\pi_{i}(M) \rightarrow G$ which vary continuously as the representations vary continuously. Furthermore, the following theorem of Van Est [14] allows one to compute $H_{\text {cont }}^{*}(G ; \mathbf{R})$.

THEOREM (VAN EST). If $K$ is a maximal compact subgroup of $G$, and $\mathfrak{k}, \mathfrak{g}$ denote the corresponding Lie algebras, then $H_{\text {cont }}^{*}(G ; \mathbf{R}) \simeq H^{*}(\mathfrak{g} ; \mathfrak{k} ; \mathbf{R})$, where the right-hand side denotes Lie algebra cohomology.

In particular, $H_{\text {cont }}^{*}(G: \mathbf{R})$ is finite dimensional.

One may think of the Lie algebra cohomology $H^{*}(\mathfrak{g} ; \boldsymbol{k} ; \mathbf{R})$ as being identified with the $G$-invariant differential forms on the contractible homogeneous space $G / K$. Now if $C$ is any contractible space on which $G$ acts, and $\pi_{1}(M) \rightarrow G$ is a representation, there is a natural map $H^{*}\{G$-invariant forms on $C\} \rightarrow H^{*}(M ; \mathbf{R})$, which is obtained as follows: if $\tilde{M}$ is the universal cover of $M$, then one may pull back forms on $C$ to forms on $\tilde{M} \times C$; those which are $G$-invariant will descend to forms on $\tilde{M} \times C / \pi_{1}(M)$, which we may regard as a flat bundle over $M$ with fiber $C$. The 
composition $(G$-invariant forms on $C) \rightarrow$ forms on $\tilde{M} \times C / \pi_{1}(M)$ clearly commutes with $d$. Since $C$ is contractible, we may identify $H^{*}\left(\tilde{M} \times C / \pi_{1}(M)\right) \cong H^{*}(M)$, yielding the desired map.

Dupont [4] gave a lovely description of this map on the chain level. (His description was also noted by others.) Indeed, let $f_{t}$ be a contraction of $C$ to a point $p \in C$, and let $\varphi$ be a $G$-invariant form on $C$. To evaluate $\varphi$ on $\left(g_{1}, \ldots, g_{k}\right) \in G^{k}$, we form the "geodesic simplex" $\Delta\left(g_{1}, \ldots, g_{k}\right)$ determined by $f_{t}$ by the following inductive method: for a 1-simplex $\left(g_{1}\right)$ let $\Delta\left(g_{1}\right)=\left\{f_{t}\left(g_{1}\right): 0 \leq t \leq 1\right\}$. Assume that all $i$-simplices $\Delta\left(g_{1}, \ldots, g_{i}\right)$ have been defined. Then set

$$
\Delta\left(g_{1}, \ldots, g_{i+1}\right)=\left\{f_{t}\left(g_{1} \cdot \Delta\left(g_{1}^{-1} g_{2}, \ldots, g_{1}^{-1} g_{i+1}\right)\right): 0 \leq t \leq 1\right\} .
$$

A closed-form definition of this inductive procedure is given in [2].

As an example, let $G=\operatorname{PSL}(2, \mathbf{R})$, and $C=G / K$ be the upper half-plane. Then the only $G$-invariant form on $C$ is the non-Euclidean volume form.

A geometrically interesting contraction of $C$ to the point $i \in C$ is given by contraction along geodesic lines. It is not hard to see that the corresponding geodesic simplex $\Delta(f, g)$ is the geodesic triangle with endpoints $i, f(i)$ and $g f(i)$. The theorem of Milnor [9] (mentioned in the introduction) can now be read off from the fact that the volume of a geodesic triangle in the upper half-plane is bounded.

Note that since there is only one $G$-invariant 2 -form on $G / K$, all elements of $H_{\text {cont }}^{2}(G ; \mathbf{R})$ must agree up to a universal constant. This yields Proposition 2 , modulo evaluation of this constant. The latter can be worked out by a special example.

The following construction is useful in this context. Let $G$ be any topological group and let $G^{\delta}$ denote the group $G$ with the discrete topology. The identity map $G^{\delta} \rightarrow G$ is a continuous homomorphism. There is a topological group (the homotopy-theoretic fiber) $\bar{G}$ and a continuous homomorphism $\bar{G} \rightarrow G$ such that $\bar{G} \rightarrow G^{\delta} \rightarrow G$ is a fibration sequence. $B \bar{G}$ classifies flat $G$-bundles which are globally topologically trivial. The continuous cohomology of $\bar{G}$ is given by the Lie algebra cohomology $H^{*}(\mathfrak{g}, \mathbf{R})$.

Specializing our discussion to $G=\operatorname{PSL}(2, \mathbf{R})$, we find that there is a natural homomorphism $\left(\mathrm{SL}(2, \mathbf{R})^{\delta}\right)^{\sim} \rightarrow \bar{G}$ which is a homotopy-equivalence. The induced map $B\left(\mathrm{SL}(2, \mathbf{R})^{\delta}\right) \rightarrow B \bar{G}$ is thus a homotopy-equivalence. The continuous cohomology of $\widetilde{\mathrm{SL}}(2, \mathbf{R})$ is given by the Lie algebra cohomology of $\operatorname{sl}(2, \mathbf{R})$, which has one generator in dimension 3 , corresponding to the invariant volume form of $\widetilde{\mathrm{SL}}(2, \mathbf{R})$.

To apply these considerations to the Godbillon-Vey invariant, let $M$ have an $\mathbf{R} P^{1}$-foliation $\mathfrak{F}$, and let $h: \pi_{1}(M) \rightarrow \operatorname{PSL}(2, \mathbf{R})$ be the holonomy homomorphism. By Lemma $2, G-V(\mathfrak{F})$ depends only on this map $h$. Furthermore, the fact that the induced bundle $\left(S^{1}\right)(M)$ has a section implies that $h$ lifts to a homomorphism:

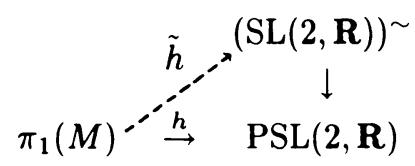

Since different choices of lifts $\tilde{h}$ correspond to different trivializations of $\left(S^{\mathbf{1}}\right) M$, it follows from Lemma 2 that $G-V(\mathfrak{F})$ is determined by a representation $\tilde{h}: \pi_{1}(M) \rightarrow$ $(\mathrm{SL}(2, \mathbf{R}))^{\sim}$. Therefore, $G-V$ is represented as a class in $H_{\text {cont }}^{3}\left((\operatorname{SL}(2, \mathbf{R}))^{\sim} ; \mathbf{R}\right)$, 
and hence is given, by Dupont's recipe, in terms of the left-invariant volume form of $(\operatorname{SL}(2, \mathbf{R}))^{\sim}$.

We will carry out this recipe in the next section.

4. An analytic estimate. We begin this section by studying the map

$$
w: \mathrm{SL}(2, \mathbf{R}) \rightarrow \mathbf{R} /(2 \pi) \mathrm{Z} \simeq S^{1},
$$

which we call the winding number, given by

$$
w\left(\begin{array}{ll}
a & b \\
c & d
\end{array}\right)=-\arg (c(i)+d)(\bmod 2 \pi) .
$$

$w$ has the following simple interpretation: if we view $\operatorname{SL}(2, \mathbf{R})$ as acting on the upper half-plane $\mathbf{H}$, then the derivative of $\left(\begin{array}{ll}a & b \\ c & d\end{array}\right)$ at $i$ is just $1 /(c i+d)^{2}$, so that $w$ measures the angular rotation of $\left(\begin{array}{ll}a & b \\ c & d\end{array}\right)$ as viewed from $i$. Note that

$$
w\left(\begin{array}{cc}
\cos (\theta) & \sin \theta \\
-\sin \theta & \cos (\theta)
\end{array}\right)=\theta
$$

In general, $w$ will not be a homomorphism, but the chain rule shows that it is almost so. To explain this, let

$$
\tilde{w}: \operatorname{SL}(2, \mathbf{R}) \times \mathbf{H} \rightarrow \mathbf{R} /(2 \pi) \mathbf{Z}
$$

be given by

$$
\tilde{w}\left(\left(\begin{array}{ll}
a & b \\
c & d
\end{array}\right), z\right)=-\arg (c z+d)(\bmod 2 \pi) .
$$

Note that since $c$ and $d$ are real and $\operatorname{Im}(z)>0$, we have that

$$
\left|\tilde{w}\left(A, z_{1}\right)-\tilde{w}\left(A, z_{2}\right)\right|<\pi
$$

for any $z_{1}, z_{2} \in \mathbf{H}$.

LEMMA 3. If $A, B \in \mathrm{SL}(2, \mathbf{R})$, then $w(A B)=\tilde{w}(A, B(i))+w(B)$.

ProOF. This is just the chain rule; alternatively, this can be verified by a simple explicit calculation.

We now lift $w$ and $\tilde{w}$ to maps

$$
w:(\mathrm{SL}(2, \mathbf{R}))^{\sim} \rightarrow \mathbf{R}, \quad \tilde{w}:(\mathrm{SL}(2, \mathbf{R}))^{\sim} \times \mathbf{H} \rightarrow \mathbf{R}
$$

which we denote by the same symbols. Lemma 3 and $(*)$ continue to hold for these maps, by analytic continuation. Furthermore, the following properties are evident.

LEMMA 4. (i) $|w(A B)-(w(A)+w(B))|<\pi$.

(ii) $\left|w\left(A B A^{-1}\right)-w(B)\right|<2 \pi$.

(iii) $\left|w\left(A B A^{-1} B^{-1}\right)\right|<2 \pi$.

ProOF. (i) follows directly from Lemma 1. To establish (ii), we write

$$
w\left(A B A^{-1}\right)=\tilde{w}\left(A, B A^{-1}(i)\right)+\tilde{w}\left(B A^{-1}(i)\right)+w\left(A^{-1}\right)
$$

together with $w\left(A^{-1}\right)=-\tilde{w}\left(A, A^{-1}(i)\right)$, to get

$$
\left|w\left(A B A^{-1}\right)-w(B)\right| \leq\left|\tilde{w}\left(A, B A^{-1}(i)\right)-\tilde{w}\left(A, A^{-1}(i)\right)\right|+\left|\tilde{w}\left(B, A^{-1}(i)\right)-w(B)\right|
$$

and (ii) then follows from (*).

(iii) follows similarly. 
We now have shown that $w$ has all the formal properties that Wood [15] used in his study of the Euler class via rotation numbers. Our main reason for introducing $w$ into the discussion is the following: we may identify $\operatorname{SL}(2, \mathbf{R}))^{\sim}$ with $\mathbf{H} \times \mathbf{R}$ by the identification $A \rightarrow(A(i), w(A))$ where we let $(\operatorname{SL}(2, \mathbf{R}))^{\sim}$ act on $\mathbf{H}$ by first projecting it onto $\operatorname{PSL}(2, \mathbf{R})$. We then have

LEMMA 5. The $(\mathrm{SL}(2, \mathbf{R}))$-invariant volume on $\mathbf{H} \times \mathbf{R}$ is given by $\left[d x \wedge d y / y^{2}\right] \wedge$ $d \tilde{w}$.

PROOF. Since $d x \wedge d y / y^{2}$ is the invariant volume on $\mathbf{H}$, it is clear that this is invariant on $\mathbf{H} \times \mathbf{R}$. $d w$, on the other hand, is not $\operatorname{PSL}(2, \mathbf{R})$-invariant. However, from the formula of Lemma 3, we write $A^{*}(d w)(B)=d \tilde{w}(A, B(i))+d w(B)$, where the first expression on the right depends only on $B(i)$, and not on $w(B)$, so that it is expressible in terms of $d x$ and $d y$. It follows that this term vanishes in the wedge product, so that $d x \wedge d y / y^{2} \wedge d w$ is invariant.

We now let $f_{t}$ be the following contraction of $\mathbf{H} \times \mathbf{R}$ to the point $(i, 0) ; f_{t}$ contracts the first factor $\mathbf{H}$ to the point $i$ along geodesic lines, while it contracts the second factor linearly. For $f_{1}, f_{2}, f_{3} \in(\mathrm{SL}(2, \mathbf{R}))^{\sim}$, let $\Delta\left(f_{1}, f_{2}, f_{3}\right)$ denote the "geodesic simplex" described above for $f_{1}, f_{2}, f_{3}$. Its vertices are the four points $\left(I, f_{1}, f_{2} f_{1}, f_{3} f_{2} f_{1}\right)$.

LEMMA 6. $\operatorname{vol}\left(\Delta\left(f_{1}, f_{2}, f_{3}\right)\right) \leq 2 \pi \max \left(w\left(f_{1}\right) w\left(f_{2}, f_{1}\right), w\left(f_{3} f_{2} f_{1}\right)\right)$.

ProOF. Projecting $\Delta\left(f_{1}, f_{2}, f_{3}\right)$ onto $\mathbf{H}$ yields a geodesic quadrilateral in $\mathbf{H}$, whose area is thus at most $2 \pi$, while projecting $\Delta\left(f_{1}, f_{2}, f_{3}\right)$ onto $\mathbf{R}$ yields an interval of length $\left\{\max \left(w\left(f_{1}\right), w\left(f_{2} f_{1}\right), w\left(f_{3} f_{2} f_{1}\right)\right)\right\}$.

5. An estimate on the fundamental group. Let $\Pi$ be a finitely presented group, with generators $g_{1}, \ldots, g_{j}$ and relations $R_{1}, \ldots, R_{k}$.

LEMMA 7. Let $\Pi$ be a finitely presented group. There exists a function c: $\Pi \rightarrow$ $\mathbf{R}$ such that if $\psi \in \operatorname{Hom}(\Pi, \operatorname{PSL}(2, \mathbf{R}))$ lifts to a homomorphism $\Pi \rightarrow \widetilde{\mathrm{SL}}(2, \mathbf{R})$, then there exists a lift $\tilde{\psi} \in \operatorname{Hom}(\Pi, \widetilde{\mathrm{SL}}(2, \mathbf{R}))$ such that $|w(\tilde{\psi}(g))| \leq c(g)$ for all $g \in \Pi$.

PROOF. We begin by lifting each generator $g_{i}$ to an element $\tilde{g}_{i}$ of $(\operatorname{SL}(2, \mathbf{R}))^{\sim}$ with winding number between 0 and $\pi$. In general, this will not extend to a representation $\Pi \rightarrow(\operatorname{SL}(2, \mathbf{R}))^{\sim}$, because the relations $R_{j}$ may go to a lift of the identity element with winding number $\pi l_{j}$.

However, by Lemma $4(\mathrm{i}), l_{i}$ is bounded in terms of the length of the word $R_{i}$, since the winding number of each $g_{i}$ is between 0 and $\pi$. Of course, using Lemma 4(ii) and (iii), one may improve upon this estimate in specific cases.

We now consider what happens when we choose a different lifting of the $g_{i}$ 's. Let $\varsigma$ denote the generator of the center of $\widetilde{\mathrm{SL}}(2, \mathbf{R})$ with $w(\varsigma)=\pi$. The lifts of the identity in $\operatorname{PSL}(2, \mathbf{R})$ are the powers $\zeta^{k}, k \in \mathbf{Z}$; thus the different lifts of $g_{i}$ to $\widetilde{\mathrm{SL}}(2, \mathbf{R})$ are obtained by multiplying the given lift $\tilde{g}_{i}$ with $\zeta^{k_{i}}$ and have winding numbers $\tilde{w}\left(\tilde{g}_{i} \varsigma^{k_{i}}\right)=\tilde{w}\left(\tilde{g}_{i}\right)+k_{i} \pi$.

Let $m_{i j}$ be the sum of the exponents of $g_{i}$ in the relation $R_{j}$. Then choosing a lift $\tilde{g}_{i} \zeta^{k}$ instead of $\tilde{g}_{i}$ adds $k_{i} m_{i j} \pi$ to the winding number of $R_{j}$. A lifting of $\psi$ to $(\operatorname{SL}(2, \mathbf{R}))^{\sim}$ will then be found by solving the system of linear equations $\sum_{i} k_{i} m_{i j}=-l_{j}$ for integers $k_{i}$. 
By the assumption that the representation lifts to $\widetilde{\mathrm{SL}}(2, \mathbf{R})$, there is a solution to this system of linear equations. Since the $l_{j}$ 's are bounded independent of $\psi$, there are only finitely many possible such systems of linear equations. Hence, there is a bound, independent of $\psi$, on the size of the $k_{i}$ 's.

Denoting by $\tilde{\psi}$ the representation resulting from this choice of $k_{i}$, it follows that

$$
\left|\left(\tilde{\psi}\left(g_{i}\right)\right)\right| \leq\left(1+\left|k_{i}\right|\right) \pi
$$

so that $\left|w\left(\tilde{\psi}\left(g_{i}\right)\right)\right|$ is bounded independent of $\psi$. A bound for $|w(g)|, g \in \Pi$, now follows from this, again by Lemma $2(\mathrm{i})$, by expressing $g$ as a word in the $g_{i}$ 's.

As a simple example, let $\Pi$ be the fundamental group of a circle bundle over a surface $S$, of genus $g>1$, with Euler class $e$. Then a presentation of $\Pi$ is given by

$$
\Pi=\left\{X_{i}, Y_{i}, T ; T^{e}=\prod_{i=1}^{g}\left(X_{i} Y_{i} X_{i} Y_{i}^{-1}\right), X_{i} T X_{i}^{-1}=T, Y_{i} T Y_{i}^{-1} T\right\} .
$$

Now the $X_{i}$ 's and $Y_{i}$ 's each occur in these relations with total degree 0 , and the winding number of a commutation is $\leq 2 \pi$, so we must solve the linear equation

$$
e k_{t}=l, \quad \text { where }|l| \leq 2 g .
$$

In particular, $\left|k_{t}\right|=|l / e| \leq 2 g /|e|$.

6. An estimate on the Godbillon-Vey class. We now return to the main situation of the paper. Suppose that $M$ has an $\mathbf{R} P^{1}$-foliation $\mathfrak{F}$. Let $h: \pi_{1}(M) \rightarrow$ $\operatorname{PSL}(2, \mathbf{R})$ be the associated holonomy homomorphism, and $\left(S^{1}\right) M$ the associated flat circle bundle over $M$.

From our remarks in $\S 3$, we may lift $h$ to a representation

$$
\tilde{h}: \pi_{1}(M) \rightarrow(\operatorname{SL}(2, \mathbf{R}))^{\sim},
$$

and $G-V(\mathfrak{F})$ may be computed from $\tilde{h}$, via Dupont's recipe, by identifying $G-V$ with the left-invariant volume form on $(\mathrm{SL}(2, \mathbf{R}))^{\sim}$.

At this point, we may replace $M$ by $K\left(\pi_{1}(M), 1\right)$, since the natural map

$$
H_{\text {cont }}^{*}(\mathrm{SL}(2, \mathbf{R})) \rightarrow H^{*}(M)
$$

factors through $H^{*}\left(K\left(\pi_{1}(M), 1\right)\right)$.

We claim that the Godbillon-Vey class in $H^{*}\left(K\left(\pi_{1}(M), 1\right)\right)$ is bounded in terms of $\pi=\pi_{1}(M)$. To see this, let $\eta$ represent any homology class in $H_{3}(K(\pi, 1)) . \eta$ is represented by a certain number of simplices in the Eilenberg-Mac Lane homology of $\pi$, say $\eta=\sum a_{i}\left(g_{i_{1}}, g_{i_{2}}, g_{i_{3}}\right)$.

By $\S 4$, we may choose our lift from $\pi \rightarrow \operatorname{PSL}(2, \mathbf{R})$ to $\pi \rightarrow \operatorname{SL}(2, \mathbf{R})$ so that the winding numbers of each $g_{i}$ is bounded by a constant $C_{i_{j}}$ depending only on $g_{i_{j}}$ and not on the representation (a different lift corresponds to a different trivialization of our circle bundle). It follows that the volume of each simplex, and hence the Godbillon-Vey class evaluated on that simplex, is bounded. It follows that the value of the Godbillon-Vey class on $\eta$ is bounded in terms of $\pi$, and independent of the representation.

It turns out that in particular cases, covering space arguments allow us to obtain sharper estimates:

THEOREM 4. Let $M$ be the total space of a circle bundle with Euler class $e$ over a closed surface $S$ of genus $g>1$, and $\mathfrak{F}$ and $\mathbf{R} P^{1}$-foliation of $M$. 
(a) If $e=0$, then $G-V(\mathfrak{F})=0$.

(b) If $e \neq 0$, then

$$
|G-V(\mathfrak{F})[M]| \leq(\text { const })\left|\chi^{2}(S) / e\right| .
$$

ProOF. Recall the presentation of the fundamental group of $M$ given in $\S 5$.

To establish (a), we note first that the above discussion gives a bound $|G-V(\mathfrak{F})[M]| \leq C$ for some $C$. For each $k$, let $\pi(k)$ denote the subgroup of $\pi_{1}(M)$ generated by $X_{i}, Y_{i}, T^{k} ; \pi_{1}(M)$ is isomorphic to $\pi(k)$, and so gives rise to a $k$-sheeted covering $\theta(k): M \rightarrow M$.

In particular,

$$
k|G-V(\mathfrak{F})|=\mid G-V\left(\theta(k)^{*}(\mathfrak{F}) \mid \leq C,\right.
$$

so that $|G-V(\mathfrak{F})| \leq C / k$. Letting $k \rightarrow \infty$ establishes (a).

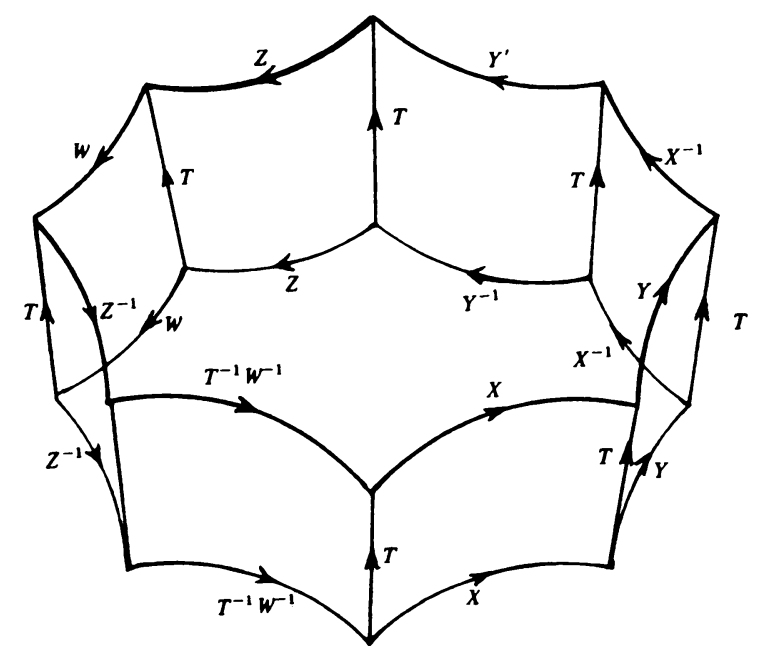

FIGURE 1

To establish (b), it suffices to consider the case $e=1$. Indeed, replacing $T$ by $T^{e}$ gives rise to an $|e|-$ fold covering of $M$; so that $G-V$ also gets multiplied by $|e|$. Since $|e|$ occurs in the denominator of our bound, we need only show it in this case.

Suppose $e=1$. Then there exists a triangulation of $M$ with $6(g+1)$ simplices: the standard triangulation of a surface has $2 g+2$ simplices, and over the cut-open surface the bundle trivializes (Figure 1); we may then erect a prism over each 2simplex, and then each prism can be divided into three simplices. (To check that the triangulations of the prisms can be chosen mutually compatible along vertical faces, one must verify a simple combinatorial lemma, left to the reader.) Figure 2 demonstrates how to express these simplices as Eilenberg-Mac Lane simplices on the fundamental group.

Now, by our computation of $\S 5$, the winding number of $T$ is at most $2 \pi g$, while the winding numbers of the other generators are at most $\pi$. It follows from $\S 4$ that

$$
|G-V(\mathfrak{F})[M]| \leq \text { (const) } g[6(2 g-1)]
$$

for some constant.

To achieve the slightly sharper results of the theorem, we may lift this estimate via $k$-fold coverings of the surface $S$; which multiply $g-1, e$ and $G-V(\mathfrak{F})$ by $k$. 
The value of (const) given here is not sharp. We will see in $\S 7$ how to obtain the sharp value (const) $=4 \pi^{2}$.

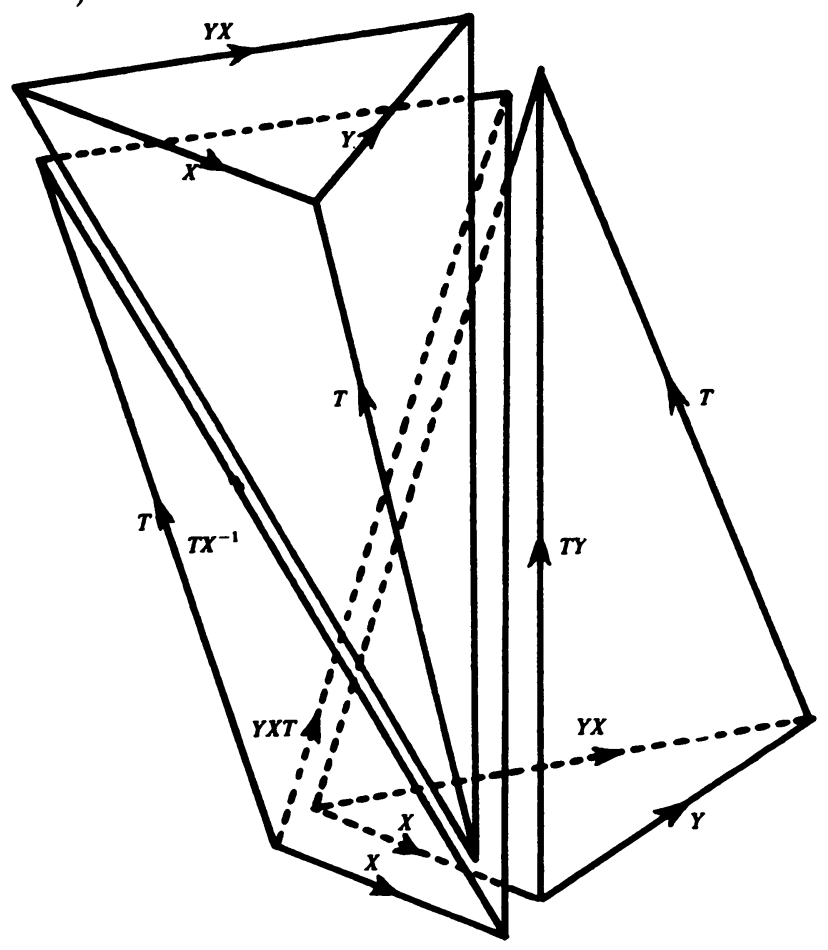

Figure 2

7. An improved bound. In this section, we will improve the bound of Theorem 4 for circle bundles over surfaces, to get the sharp bound:

THEOREM 5. Let $\mathfrak{F}$ be a real projective foliation of $M$, a circle bundle with Euler class $e \neq 0$ over a closed surface $S$ of genus $g>1$. Then

$$
|G-V(\mathfrak{F})| \leq\left(4 \pi^{2}\right)\left|\chi^{2}(S) / e\right|
$$

and $(G-V(\mathfrak{F})) / 4 \pi^{2}$ is an integer.

PROOF. Let $h: \pi_{1}(M) \rightarrow \operatorname{PSL}(2, \mathbf{R})$ denote a holonomy homomorphism for $\mathfrak{F}$ and $\tilde{h}: \pi_{1}(M) \rightarrow \operatorname{SL}(2, \mathbf{R})^{\sim}$ be a lift. Let $T \in \pi_{1}(M)$ be a central element corresponding to the fiber.

Claim. If $G-V(\mathfrak{F}) \neq 0$, then $\tilde{h}(T)=\varsigma^{k}$ for some $k \in \mathbf{Z}-\{0\}$.

Proof. Suppose $h(T) \neq 1$. Since $T$ is central in $\pi_{1}(M), h(\Pi)$ must lie in the centralizer $C$ of $h(T)$, which is a one-dimensional connected Lie group. We may then take $\tilde{h}$ to factor through $\tilde{C}$, and the map $H_{\text {cont }}^{3}\left((\operatorname{SL}(2, \mathbf{R}))^{\sim}\right) \rightarrow H^{3}(M)$ to factor through $H_{\text {cont }}^{3}(\tilde{C})$, which is 0 . It follows that $G-V(\mathfrak{F})=0$.

Thus $\tilde{h}(T)=\varsigma^{k}$ for some $k \in \mathbf{Z}$. If $k=0$, then $h$ factors as $\pi_{1}(M) \rightarrow \pi_{1}(S) \rightarrow$ $\operatorname{PSL}(2, \mathbf{R})$. Since $H^{3}(S)=0, G-V(\mathfrak{F})=0$.

In general, the integer $k$ has a simple interpretation as follows. Since $h(T)=1$, the developing map factors through a submersion $\delta: \hat{M} \rightarrow \mathbf{R} P^{1}$, where $\hat{M}$ is a covering space of $M$ such that $T$ lifts to a closed loop $T$ in $M$. Then $k$ equals the 
degree of $\delta: T \rightarrow \mathbf{R} P^{1}$. In particular, $k=1$ if and only if $h$ is the holonomy of a flat $\mathbf{R} P^{1}$-bundle over $S$.

Let $M(k)$ denote the total space of a circle bundle over $S$ with Euler class $k \cdot e$. Then there is a $k$-fold covering $p: M \rightarrow M(k)$. Furthermore since $\tilde{h}(T)=\varsigma^{k}$, there exists a homomorphism $h: \pi_{1}(M(k)) \rightarrow \operatorname{SL}(2, \mathbf{R})$ such that $\tilde{h}: \pi_{1}(M) \rightarrow \widetilde{\mathrm{SL}}(2, \mathbf{R})$ factors as $\pi_{1}(M) \stackrel{p}{\rightarrow} \pi_{1}(M(k)) \stackrel{h^{\prime}}{\rightarrow} \widetilde{\mathrm{SL}}(2, \mathbf{R})$.

The theorem of Milnor and Wood now implies that $|e \cdot k| \leq|\chi(S)|$. From Proposition 2, we also calculate $G-V\left(h^{\prime}\right)=4 \pi^{2} e \cdot k$. Passing to a $k$-fold cover gives $G-V(\mathfrak{F})=4 \pi^{2} e \cdot k^{2}$ and so $|G-V(\mathfrak{F})| \leq 4 \pi^{2}\left|\chi^{2}(S) / e\right|$ as desired.

\section{REFERENCES}

1. R. Blumenthal, Thansversely homogeneous foliations, Ann. Inst. Fourier (Grenoble) 29 (1979), 143-158

2. R. Brooks, On the smooth cohomology of groups of diffeomorphisms, Ph.D. thesis, Harvard University, 1977.

3. _ Volumes and characteristic classes of foliations, Topology 18 (1979), 295-304.

4. J. L. Dupont, Simplicial deRham cohomology and characteristic classes of flat bundles, Topology 15 (1976), 233-246.

5. C. Godbillon and J. Vey, Un invariant des feuilletages de codimension un, C.R. Acad. Sci. Paris Sér. I. Math. 273 (1971), 92.

6. W. Goldman, Discontinuous groups and the Euler class, Ph.D. thesis, University of California, Berkeley, 1980.

7. M. Gromov, Volume and bounded cohomology, Inst. Hautes Études Sci. Publ. Math. 56 (1982), 5-99.

8. J. Heitsch, Secondary invariants of transversely homogeneous foliations, preprint.

9. J. Milnor, On the existence of a connection with curvature zero, Comment. Math. Helv. 32 (1958), 215-223.

10. D. Sullivan, A generalization of Milnor's inequality concerning affine foliations and affine manifolds, Comment. Math. Helv. 51 (1976), 183-199.

11. W. P. Thurston, Foliations and groupes of diffeomorphisms, Bull. Amer. Math. Soc. 80 (1974), 304-312.

12. , Non-cobondant foliations of $S^{3}$, Bull. Amer. Math. Soc: 78 (1972), 511-514.

13. __ Foliations of three-manifolds which are circle bundles, Ph.D. thesis, University of California, Berkeley, 1972.

14. W. Van Est, Une application d'une méthode de Cartan-Leray, Indag. Math. 17 (1955), 542-544.

15. J. Wood, Foliated $S^{1}$-bundles and diffeomorphioms of $S^{1}$, Dynamical Systems (M. Peixoto, ed.), Academic Press, New York, 1973.

16. H. Blaine Lawson, Fotiations, Bull. Amer. Math. Soc. 80 (1974), 369-418.

17. R. Bott, On the characteristic classes of group actions, Lecture Notes in Math., vol. 652, SpringerVerlag, Berlin and New York, 1978, pp. 26-61.

18. M. Hirsch and W. Thurston, Foliated bundles, invariant measures, and flat manifolds, Ann. of Math. (2) 101 (1975), 369-390.

19. C. Chevalley and S. Eilenberg, Cohomology theory of Lie groups and Lie algebras, Trans. Amer. Math. Soc. 63 (1948), 85-124.

20. R. Brooks and W. Goldman, Volumes in Seifert space, Duke Math J. (to appear).

21. W. Thurston, Three-dimensional manifolds, Kleinian groups, and hyperbolic geometry, Bull. Amer. Math. Soc. 6 (1982), 357-382.

Courant institute of Mathematical SCIENCES, New YORK UNiversity, NeW YORK, NEW YORK 10012

Department of Mathematics, Massachusetts institute of Technology, CAMBRIDGE, MASSACHUSETTS 02139 (Current address of William Goldman)

Current address (R. Brooks): University of Southern California, Los Angeles, California 90089-1113 